\title{
IgG4-positive plasma cell infiltration in the diagnosis of autoimmune pancreatitis
}

\author{
Lizhi Zhang ${ }^{1}$, Kenji Notohara ${ }^{2}$, Michael J Levy ${ }^{3}$, Suresh T Chari ${ }^{3}$ and Thomas C Smyrk ${ }^{1}$ \\ ${ }^{1}$ Department of Laboratory Medicine and Pathology, Mayo Clinic, Rochester, MN, USA; ${ }^{2}$ Department of \\ Pathological Research, Okayama University Graduate School of Medicine and Dentistry, Okayama, Japan \\ and ${ }^{3}$ Division of Gastroenterology and Hepatology, Department of Internal Medicine, Mayo Clinic, Rochester, \\ $M N, U S A$
}

\begin{abstract}
Autoimmune pancreatitis typically produces an enlarged pancreas with narrowing of the pancreatic duct, and can mimic carcinoma. Autoimmune pancreatitis usually responds to corticosteroid treatment, making it important to differentiate from pancreatic ductal adenocarcinoma. Affected patients often have an elevated serum IgG4. It has been proposed that increased numbers of IgG4-positive plasma cells in tissue might be a marker for the condition. We investigated the role of IgG4 staining in the diagnosis of autoimmune pancreatitis, first in resected pancreas specimens (29 autoimmune pancreatitis, nine chronic alcoholic pancreatitis and 25 pancreatic cancer), then in pancreatic needle biopsies. Immunohistochemical stains for IgG4 were scored as none, mild, moderate or marked, according to published criteria. Moderate to marked numbers of IgG4-positive plasma cells were seen in 21/29 autoimmune pancreatitis patients, and were distributed in and around ducts, in interlobular fibrous tissue and in peripancreatic fat. In contrast, eight of nine examples of chronic alcoholic pancreatitis and 22/25 ductal adenocarcinomas had scores of none or mild. When we subdivided autoimmune pancreatitis into the histologic subtypes lymphoplasmacytic sclerosing pancreatitis and idiopathic ductdestructive pancreatitis, 16/17 lymphoplasmacytic sclerosing pancreatitis had moderate to marked staining, compared to five to 12 idiopathic duct-destructive pancreatitis. Needle biopsies from nine patients suspected of having autoimmune pancreatitis had increased numbers of IgG4 cells. We conclude that pancreatic tissue from patients with autoimmune pancreatitis often shows moderate or marked infiltration by IgG4-positive plasma cells (>10/HPF). This is particularly so in the subtype we have designated lymphoplasmacytic sclerosing pancreatitis. We rarely see IgG4 staining in patients with chronic alcoholic pancreatitis and pancreatic ductal adenocarcinoma. IgG4-positive plasma cells are a useful marker for the tissue diagnosis of autoimmune pancreatitis.

Modern Pathology (2007) 20, 23-28. doi:10.1038/modpathol.3800689; published online 15 September 2006
\end{abstract}

Keywords: autoimmune pancreatitis; IgG4; chronic pancreatitis; pancreatic ductal adenocarcinoma; immunohistochemistry

Autoimmune pancreatitis is a rare form of chronic pancreatitis, first described in 1961 as 'primary inflammatory sclerosis of the pancreas'. ${ }^{1}$ Subsequent reports have used various names, including lymphoplasmacytic sclerosing pancreatitis, chronic sclerosing pancreatitis, nonalcoholic duct destructive chronic pancreatitis and inflammatory pseudotumor. $^{2-6}$ Autoimmune pancreatitis is the currently preferred term because clinical, serologic, histological and immunohistochemical findings suggest an autoimmune mechanism. Autoimmune pancreatitis has been described in association with other auto-

Correspondence: Dr TC Smyrk, MD, Department of Laboratory Medicine and Pathology, Mayo Clinic, 200 First Street SW, Rochester, MN 55905, USA.

E-mail: smyrk.thomas@mayo.edu

Received 20 June 2006; revised 3 August 2006; accepted 4 August 2006; published online 15 September 2006 immune disorders such as Sjogren syndrome, primary sclerosing cholangitis, idiopathic retroperitoneal fibrosis and inflammatory bowel disease. ${ }^{7-13}$ But 'association' may be a misleading term; it has been argued that autoimmune pancreatitis is a systemic disease that can produce, for example, a Sjogren-like salivary gland enlargement and a primary sclerosing cholangitis-like biliary disease. ${ }^{14}$ Most affected patients have hypergammaglobulinemia and increased serum levels of IgG, particularly IgG4. ${ }^{15,16}$ There also may be autoantibodies directed against lactoferrin (LF), carbonic anhydrase-II and IV (CA-II, CA-IV), rheumatoid factor, smooth muscle antigens and nuclear antigens. ${ }^{9}$ Autoimmune pancreatitis is characterized histologically by dense lymphoplasmacytic infiltration centered on pancreatic ducts, accompanied by obliterative phlebitis, acinar atrophy and interstitial fibrosis. ${ }^{6,11,17,18} \mathrm{Im}-$ munohistochemical typing reveals a predominance 
of CD8 + and CD4 + T lymphocytes, with few B lymphocytes. ${ }^{19}$ Autoimmune pancreatitis responds to steroid therapy. ${ }^{20,21}$

Autoimmune pancreatitis can mimic pancreatic cancer clinically. The presentation almost always features jaundice, and there may be abdominal pain and weight loss. Imaging usually shows diffuse enlargement of the pancreas, but there can be tumorlike local swelling. The pancreatic duct is diffusely or segmentally narrowed. Until recently, almost all autoimmune pancreatitis was diagnosed in patients undergoing pancreaticoduodenectomy for presumed pancreatic cancer. ${ }^{22,23}$ As the condition often responds to steroid treatment, a correct preoperative diagnosis is highly desirable. Despite growing awareness of the condition, differentiating autoimmune pancreatitis from cancer remains a challenge, particularly for patients with radiologic evidence of a tumefactive lesion.

Elevated serum levels of IgG4 provide a possible marker for the disease. The test characteristics have not been evaluated extensively, particularly in western populations. ${ }^{15,16}$ It is clear although, that neither the sensitivity nor the specificity is $100 \%$. Our experience shows that serum IgG4 is elevated in $70 \%$ of patients with autoimmune pancreatitis, ${ }^{24}$ a result replicated in a recent abstract showing that two of 10 lymphoplasmacytic sclerosing pancreatitis patients had normal Ig4 serum levels. ${ }^{25}$ More importantly, occasional pancreatic cancer patients can have elevated serum IgG4 levels (unpublished data). Elevated IgG4 levels have been described in atopic dermatitis, asthma, some parasitic diseases, pemphigus vulgaris and pemphigus foliaceus. ${ }^{26-29}$

Kamisawa $^{30}$ have argued that IgG4-positive plasma cell infiltration in pancreas has diagnostic utility in differentiating autoimmune pancreatitis from pancreatic cancer and from other types of chronic pancreatitis. In this study, we investigated the usefulness of immunohistochemical staining for IgG4 in pancreatic tissue, first in a series of resected pancreas, then in a series of pancreas biopsies.

\section{Materials and methods}

\section{Case Selection}

Twenty-nine resected pancreas specimens met both clinical and histologic criteria for autoimmune pancreatitis and had paraffin-embedded material available for immunohistochemical staining. The cases were collected between 1985 and 2002 . This set was described previously. ${ }^{6}$ They were divided into lymphoplasmacytic sclerosing pancreatitis $(n=17)$ and idiopathic duct-destructive pancreatitis $(n=12)$ according to the previous study. ${ }^{6}$ Briefly, lymphoplasmacytic sclerosing pancreatitis tends to affect middle-aged men and features dense periductal lymphocytic inflammation without duct epithelial damage. Idiopathic duct-destructive pan- creatitis has a broader age distribution and affects both sexes equally; duct epithelium is frequently infiltrated by neutrophils and there is epithelial damage.

Nine pancreases resected for advanced chronic alcoholic pancreatitis and 25 resected pancreases with ductal adenocarcinoma were used as controls. During the years 1999 to 2004, nine patients who were ultimately given a diagnosis of autoimmune pancreatitis had needle biopsy of the pancreas as part of their clinical workup. In two patients (patients 8 and 9), IgG4 stain was used as part of the prospective evaluation. For the other seven patients, IgG4 staining was performed retrospectively.

\section{Immunohistochemistry}

Pancreatic tissue was fixed in $10 \%$ buffered formalin and embedded in paraffin. Monoclonal antihuman IgG4 antibody (Zymed, San Francisco, CA, USA) was applied to $4-\mu \mathrm{m}$ sections using standard immunohistochemical techniques. Peroxidase activity was visualized by applying diaminobenzidine solution containing $0.05 \% \quad \mathrm{H}_{2} \mathrm{O}_{2}$. Sections were then counterstained with hematoxylin, dehydrated, cleared and mounted. Appropriate positive and negative controls were run with each batch. The extent of IgG4-positive plasma cell infiltration was evaluated independently by two pathologists (LZ, TCS). The extent was scored as none, mild, moderate or marked according to the number of immunohistochemically identified IgG4-positive plasma cells per high-power field (HPF) in each specimen (Nikon E 600, field diameter $0.625 \mathrm{~mm}$ ). The areas with highest density of IgG4-positive plasma cells were evaluated. Three HPFs per tissue section were selected and an average number of IgG4-positive plasma cells per HPF were calculated. As outlined by Kamisawa, ${ }^{30}$ tissue with less than five positive cells/HPF were scored as none, five to 10 cells led to a score of mild, 11-30 cells generated a score of moderate and tissues with more than 30 positive cells/HPF were scored as marked.

\section{Results}

IgG4-positive plasma cells were numerous in resected autoimmune pancreatitis. There was moderate to marked staining in 21 of 29 cases, compared to one of nine among alcoholic pancreatitis cases and three of 25 resected ductal adenocarcinomas (Table 1). The distribution of IgG4-positive plasma cells is illustrated in Figure 1a-d. Staining was diffuse, but tended to be accentuated in and around interlobular and intralobular ducts. Inflamed acini and peripancreatic lymphoid aggregates also contained positive cells. Fibrotic areas were the least likely to harbor IgG4-positive cells. 
Table 1 IgG4+ plasma cell infiltration in AIP, CAP and PA

\begin{tabular}{lccc}
\hline $\begin{array}{l}\text { IgG4+ plasma } \\
\text { cell infiltration }\end{array}$ & $\begin{array}{c}A I P \\
(\mathrm{n}=29)\end{array}$ & $\begin{array}{c}C A P \\
(\mathrm{n}=9)\end{array}$ & $\begin{array}{c}P A \\
(\mathrm{n}=25)\end{array}$ \\
\hline None & 1 & 4 & 16 \\
Mild & 7 & 4 & 6 \\
Moderate & 8 & 1 & 2 \\
Marked & 13 & 0 & 1 \\
Moderate-marked (total) & $21 / 29$ & $1 / 9$ & $3 / 25$ \\
& $(72.4 \%)$ & $(11.1 \%)$ & $(12 \%)$ \\
\hline
\end{tabular}

AIP, autoimmune pancreatitis; CAP, chronic alcoholic pancreatitis; PA, pancreatic ductal adenocarcinoma.

Most of the resections for chronic alcoholic pancreatitis had no or few IgG4-positive cells (Figure 1e-f). One case in this category had moderate numbers of stained cells, predominantly localized to lymphoid aggregates. Lymphoid aggregates are a pathological feature of autoimmune pancreatitis more than alcoholic pancreatitis, but other areas showed dense fibrosis without inflammation and there was a single abscess. The overall histology and the clinical history of alcohol abuse led us to classify the case as alcoholic pancreatitis. Three of 25 resected pancreatic ductal adenocarcinomas showed moderate to marked numbers of IgG4-positive cells, but the distribution of IgG4positive cells was patchy; large expanses of tissue had no positive cells. The focal collections of IgG4positive cells were seen either randomly distributed in the stroma (two cases) of infiltrating carcinoma or were in peripancreatic lymphoid aggregates (one case). There was no increased staining in areas of chronic pancreatitis adjacent to the tumor. The majority of pancreatic ductal adenocarcinomas and peritumoral pancreatic tissue had IgG4 scores of none or mild, despite the fact that some of these cases were selected for their dense peritumoral lymphocytic infiltrates (Figure 1g-h).

Using previously published criteria to subdivide autoimmune pancreatitis, ${ }^{6}$ we had 17 examples of lymphoplasmacytic sclerosing pancreatitis and 12 of idiopathic duct-destructive pancreatitis. As shown in Table 2, lymphoplasmacytic sclerosing pancreatitis was much more likely to have moderate or marked numbers of IgG4-positive cells (16/17) than was idiopathic duct-destructive pancreatitis $(5 / 12)$.

The clinical features of the nine patients who had needle biopsies prior to a diagnosis of autoimmune pancreatitis are shown in Table 3 . All biopsies had at least mild numbers of IgG4-positive cells. Patient 2 , a 50-year-old female, presented with a stricture of the pancreatic duct in the setting of known ulcerative colitis. Duct brushing cytology was suspicious for adenocarcinoma. Pylorus-sparing pancreatoduodenectomy was undertaken, but no malignancy was identified. In retrospect, this might represent the idiopathic duct-destructive pancreatitis subset of autoimmune pancreatitis. Patient 7 , a 56-year-old female, is being managed with steroids for presumed autoimmune pancreatitis despite normal serum IgG4 levels and mild IgG4-positive cell infiltrates in the needle biopsy. These two could represent the idiopathic duct-destructive pancreatitis subset of autoimmune pancreatitis. The other seven patients all have characteristic features of autoimmune pancreatitis (lymphoplasmacytic sclerosing pancreatitis subtype), except for patient 8, who was only 15 years old at the time of diagnosis. He underwent open exploration with biopsy, at which time the diagnosis of autoimmune pancreatitis was made.

\section{Discussion}

Resected pancreatic tissue from patients with autoimmune pancreatitis is often infiltrated by IgG4-positive plasma cells; $72 \%$ of our cases had moderate to marked numbers of positive cells. There was significant IgG4 staining associated with three of 25 ductal adenocarcinomas and one of nine resected examples of chronic alcoholic pancreatitis. We suggest that the IgG4 stain is useful for differentiating between autoimmune pancreatitis and other forms of chronic pancreatitis and between autoimmune pancreatitis and pancreatic ductal adenocarcinoma, but we emphasize that the finding of many ( $>10 / \mathrm{HPF}$ ) IgG4-positive cells is not $100 \%$ specific for autoimmune pancreatitis.

In a previous study, we identified two histologic subgroups in autoimmune pancreatitis, designated lymphoplasmacytic sclerosing pancreatitis and idiopathic duct-destructive pancreatitis. ${ }^{6}$ Although there is histologic overlap between two groups, idiopathic duct-destructive pancreatitis is more likely to have neutrophils in duct epithelium and to show some histologic evidence of duct epithelial damage. Zamboni et $a l^{18}$ also recognized a subtype of autoimmune pancreatitis occur in a subset of patients who are younger and more commonly have ulcerative colitis and Crohn's disease, which is characterized by the presence of granulocytic epithelial lesion. We believe this subtype is equivalent to our so-called idiopathic duct-destructive pancreatitis. Except for a tendency for lymphoplasmacytic sclerosing pancreatitis patients to be older and male sex, the two groups in our study had similar clinical presentations and courses, casting doubt on the validity of our subdivision autoimmune pancreatitis. ${ }^{31}$ The IgG4 stain, however, highlights another difference between lymphoplasmacytic sclerosing pancreatitis and idiopathic duct-destructive pancreatitis, in that 16 of 17 (94\%) specimens previously designated as lymphoplasmacytic sclerosing pancreatitis had moderate to marked numbers of IgG4-positive cells, compared to five of $12(42 \%)$ in the idiopathic duct-destructive pancreatitis group. A recent abstract by Dhall et $a 1^{25}$ supports our contention that there are two subtypes of autoimmune pancreatitis. The authors found that 

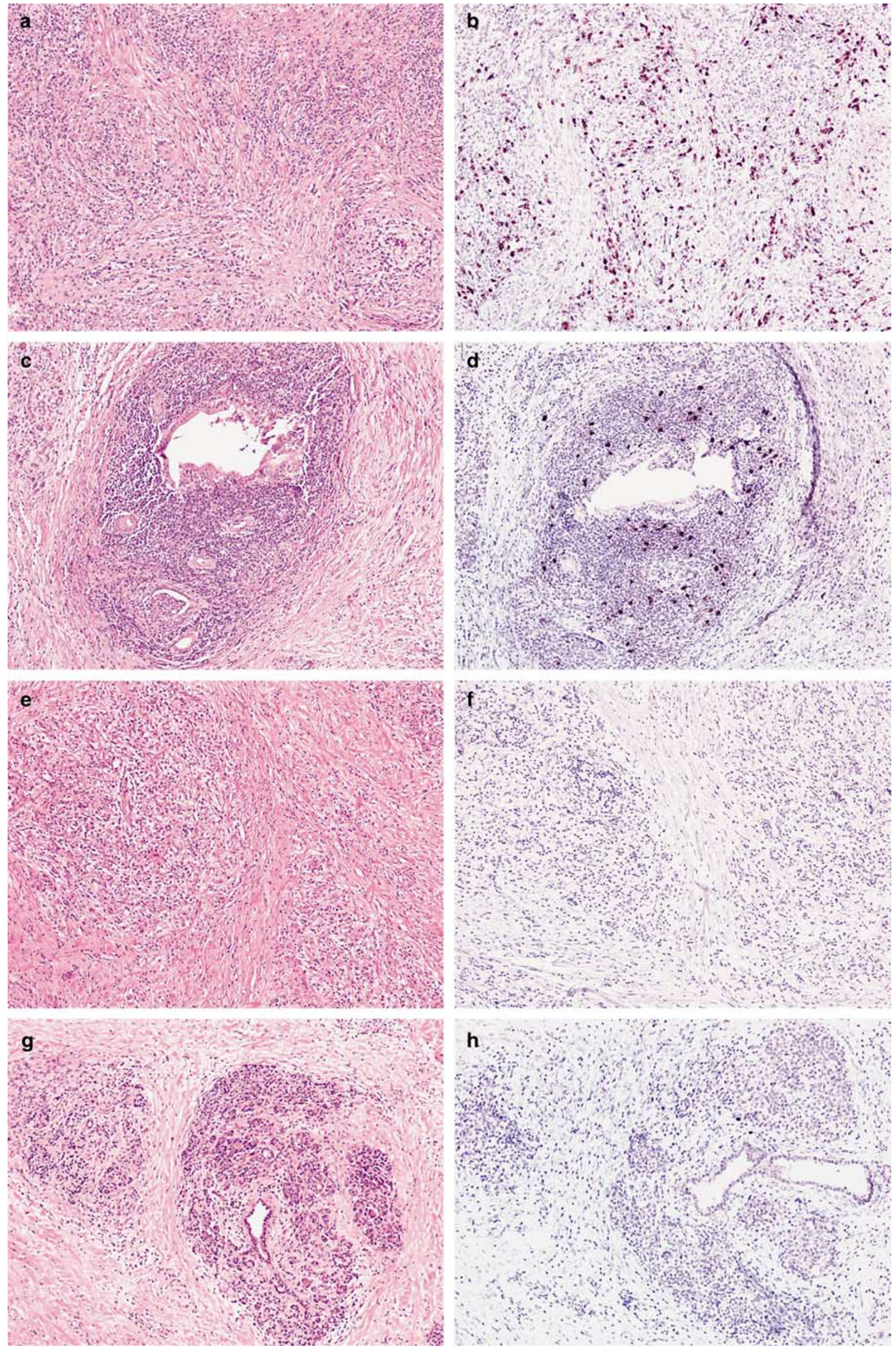
more than 50 IgG4-positive cells/HPF is highly specific for what they called 'lymphoplasmacytic sclerosing pancreatitis, usual type,' but 'lymphoplasmacytic sclerosing pancreatitis, granulocytic type' (equivalent to our idiopathic duct-destructive pancreatitis), there were many fewer IgG4-positive cells. It is still not clear whether the two subsets represent different diseases or different stages of the same disease. None of the patients in the resection group had serum IgG4 levels analyzed, and of course the fact that resections were performed precludes evaluating a response to steroids. Two patients in the series diagnosed by needle biopsy have normal serum IgG4 levels and low to moderate numbers of IgG4-positive cells, providing a potential opportunity to compare outcomes in the two putative subgroups.

Our early experience with needle biopsies is promising, but several caveats must be noted. First, this is not a prospectively evaluated series. Seven of our nine patients already had an established diagnosis of autoimmune pancreatitis on clinical and histological grounds when the IgG4 stain was evaluated. Second, our experience is limited to core biopsies obtained via ultrasound-guided endoscopy. In this setting, both the histological pattern and the IgG4 stain contribute to the diagnosis of autoimmune pancreatitis. A well-prepared cell block could have enough tissue fragments to allow a similar pattern

Table 2 IgG4+ plasma cell infiltration in LPSP and IDCP

\begin{tabular}{lcc}
\hline IgG4+ plasma cell infiltration & LPSP $(\mathrm{n}=17)$ & IDCP $(\mathrm{n}=12)$ \\
\hline None & 0 & 1 \\
Mild & 1 & 6 \\
Moderate & 4 & 4 \\
Marked & 12 & 1 \\
Moderate to marked total & $16(94.1 \%)$ & $5(41.7 \%)$ \\
\hline
\end{tabular}

IDCP, idiopathic duct-destructive pancreatitis; LPSP, lymphoplasmacytic sclerosing pancreatitis. analysis, but we have no experience with that kind of material. Applying the IgG4 stain to a cytospin preparation seems to us to be ill-advised. The histological pattern is lost and, more importantly, the concentrating effect of the preparation invalidates the proposed scoring criteria for the IgG4 stain.

The pathogenesis of autoimmune pancreatitis remains unknown. Both humoral and cellular immunity has been proposed to play a role in developing autoimmune pancreatitis, but neither the target antigen(s) nor the effector cells have been identified. Increased serum IgG4 concentrations have been documented in autoimmune pancreatitis. ${ }^{15}$ IgG4 is the least prevalent of the IgG subclasses; it is characterized by an inability to fix C1q complement and a low affinity for target antigens. IgG4-secreting plasma cells derive from antigen stimulation of naive or memory B cells in secondary lymphoid tissues such as spleen or lymph nodes. Kamisawa et $a l^{14}$ have shown that patients with autoimmune pancreatitis have IgG4-positive plasma cell infiltrations in other organs besides pancreas, including peripancreatic soft tissue, lymph nodes and bone marrow. This diffusely distributed population of IgG4-positive plasma cells may explain the increased serum IgG4 concentrations seen in many autoimmune pancreatitis patients.

Although there were three pancreatic ductal adenocarcinomas with moderate to marked IgG4positive plasma cell infiltrates, the distribution of these positive cells was different from autoimmune pancreatitis. Two cases had scattered clusters of positive cells in stroma of infiltrating carcinoma, and one had moderate numbers of positive cells in extrapancreatic lymphoid aggregates, patterns different from the diffuse and dense periductal IgG4positive plasma cell infiltrates in autoimmune pancreatitis. Some of theses cases with prominent obstructive chronic pancreatitis also showed absence of moderate or marked IgG4-positive plasma cell infiltration.

Table 3 Clinicopathologic features of patients undergoing needle biopsy of the pancreas for suspected autoimmune pancreatitis

\begin{tabular}{llllll}
\hline & Age/sex & Chief complaint & Imaging & Serum IgG4 level & Tissue IgG4+ cells \\
\hline 1 & $79 \mathrm{M}$ & Abdominal pain & $3 \mathrm{~cm}$ mass & Not done & Marked \\
2 & $50 \mathrm{~F}$ & Jaundice & Stricture & Not done & Mild \\
3 & $65 \mathrm{M}$ & Jaundice & $5 \mathrm{~cm}$ mass & Normal & Moderate \\
4 & $72 \mathrm{M}$ & Jaundice & Vague mass & Not done & Moderate \\
5 & $72 \mathrm{~F}$ & Weight loss & $3 \mathrm{~cm}$ mass & Not done & Marked \\
6 & $72 \mathrm{M}$ & Weight loss & Diffuse swelling & Elevated & Marked \\
7 & $56 \mathrm{~F}$ & Jaundice & Diffuse swelling & Normal & Mild \\
8 & $15 \mathrm{M}$ & Anemia & Mass & Not done & Marked \\
9 & $74 \mathrm{M}$ & Jaundice & Stricture & Elevated & Marked \\
\hline
\end{tabular}

Figure 1 Immunohistochemical staining for IgG4 showing marked IgG4-positive plasma cell infiltrates in autoimmune pancreatitis but not in chronic alcoholic pancreatitis and adenocarcinoma-associated chronic pancreatitis. (a, b), lymphoplasmacytic sclerosing pancreatitis; (c, d), idiopathic duct-destructive pancreatitis; (e, f), chronic alcoholic pancreatitis; (g, h), adenocarcinoma-associated chronic pancreatitis. 
In summary, we found increased numbers of pancreatic IgG4-positive plasma cells in autoimmune pancreatitis compared to chronic alcoholic pancreatitis and pancreatic ductal adenocarcinoma. The IgG4 stain is a useful adjunct to the diagnosis of autoimmune pancreatitis, and helps differentiate autoimmune pancreatitis from other types of chronic pancreatitis and from ductal adenocarcinoma.

\section{References}

1 Sarles H, Sarles JC, Muratore R, et al. Chronic inflammatory sclerosis of the pancreas-an autonomous pancreatic disease? Am J Dig Dis 1961;6: 688-698.

2 Kawaguchi K, Koike M, Tsuruta K, et al. Lymphoplasmacytic sclerosing pancreatitis with cholangitis: a variant of primary sclerosing cholangitis extensively involving pancreas. Hum Pathol 1991;22:387-395.

3 Sood S, Fossard DP, Shorrock K. Chronic sclerosing pancreatitis in Sjogren's syndrome: a case report. Pancreas 1995;10:419-421.

4 Ectors N, Maillet B, Aerts R, et al. Non-alcoholic duct destructive chronic pancreatitis. Gut 1997;41: 263-268.

5 Wreesmann V, van Eijck CH, Naus DC, et al. Inflammatory pseudotumour (inflammatory myofibroblastic tumour) of the pancreas: a report of six cases associated with obliterative phlebitis. Histopathology 2001;38: 105-110.

6 Notohara K, Burgart LJ, Yadav D, et al. Idiopathic chronic pancreatitis with periductal lymphoplasmacytic infiltration: clinicopathologic features of 35 cases. Am J Surg Pathol 2003;27:1119-1127.

7 Yoshida K, Toki F, Takeuchi T, et al. Chronic pancreatitis caused by an autoimmune abnormality. Proposal of the concept of autoimmune pancreatitis. Dig Dis Sci 1995;40:1561-1568.

8 Kino-Ohsaki J, Nishimori I, Morita M, et al. Serum antibodies to carbonic anhydrase I and II in patients with idiopathic chronic pancreatitis and Sjogren's syndrome. Gastroenterology 1996;110:1579-1586.

9 Okazaki K, Uchida K, Ohana M, et al. Autoimmunerelated pancreatitis is associated with autoantibodies and a Th1/Th2-type cellular immune response. Gastroenterology 2000;118:573-581.

10 Fukukura Y, Fujiyoshi F, Nakamura F, et al. Autoimmune pancreatitis associated with idiopathic retroperitoneal fibrosis. AJR Am J Roentgenol 2003;181: 993-995.

11 Abraham SC, Cruz-Correa M, Argani P, et al. Lymphoplasmacytic chronic cholecystitis and biliary tract disease in patients with lymphoplasmacytic sclerosing pancreatitis. Am J Surg Pathol 2003;27:441-451.

12 Takikawa H, Takamori Y, Tanaka A, et al. Analysis of 388 cases of primary sclerosing cholangitis in Japan; Presence of a subgroup without pancreatic involvement in older patients. Hepatol Res 2004;29: 153-159.

13 Ichimura T, Kondo S, Ambo Y, et al. Primary sclerosing cholangitis associated with autoimmune pancreatitis. Hepatogastroenterology 2002;49:1221-1224.
14 Kamisawa T, Egawa N, Nakajima H. Autoimmune pancreatitis is a systemic autoimmune disease. Am J Gastroenterol 2003;98:2811-2812.

15 Hamano H, Kawa S, Horiuchi A, et al. High serum IgG4 concentrations in patients with sclerosing pancreatitis. N Engl J Med 2001;344:732-738.

16 Hirano K, Kawabe T, Yamamoto N, et al. Serum IgG4 concentrations in pancreatic and biliary diseases. Clin Chim Acta 2006;367:181-184.

17 Youssef N, Petitjean B, Bonte H, et al. Non-alcoholic duct destructive chronic pancreatitis: a histological, immunohistochemical and in-situ apoptosis study of 18 cases. Histopathology 2004;44:453-461.

18 Zamboni G, Luttges J, Capelli P, et al. Histopathological features of diagnostic and clinical relevance in autoimmune pancreatitis: a study on 53 resection specimens and 9 biopsy specimens. Virchows Arch 2004;445:552-563.

19 Kamisawa T, Funata N, Hayashi Y, et al. Close relationship between autoimmune pancreatitis and multifocal fibrosclerosis. Gut 2003;52:683-687.

20 Ito T, Nakano I, Koyanagi S, et al. Autoimmune pancreatitis as a new clinical entity. Three cases of autoimmune pancreatitis with effective steroid therapy. Dig Dis Sci 1997;42:1458-1468.

21 Kamisawa T, Yoshiike M, Egawa N, et al. Treating patients with autoimmune pancreatitis: results from a long-term follow-up study. Pancreatology 2005;5: 234-238.

22 Hardacre JM, Iacobuzio-Donahue CA, Sohn TA, et al. Results of pancreaticoduodenectomy for lymphoplasmacytic sclerosing pancreatitis. Ann Surg 2003;237: 853-858.

23 Weber SM, Cubukcu-Dimopulo O, Palesty JA, et al. Lymphoplasmacytic sclerosing pancreatitis: inflammatory mimic of pancreatic carcinoma. J Gastrointest Surg 2003;7:129-137.

24 Chari ST, Smyrk TC, Levy MJ, et al. Diagnosis of autoimmune pancreatitis: the Mayo Clinic experience. Clin Gastroenterol Hepatol 2006;4:1010-1016.

25 Dhall DSA, Shia J, Tang L, et al. Immunohistochemical detection of IgG4-positive plasma cells helps distinguish lymphoplasmacytic sclerosing pancreatitis (LPSP) from pancreatitis associated with carcinoma or other conditions. Modern Pathol 2006;19:272A.

26 Aalberse RC, Van Milligen F, Tan KY, et al. Allergenspecific IgG4 in atopic disease. Allergy 1993;48: 559-569.

27 Rock B, Martins CR, Theofilopoulos AN, et al. The pathogenic effect of IgG4 autoantibodies in endemic pemphigus foliaceus (fogo selvagem). N Engl J Med 1989;320:1463-1469.

28 Bhol K, Mohimen A, Ahmed AR. Correlation of subclasses of IgG with disease activity in pemphigus vulgaris. Dermatology 1994;189:85-89.

29 Ding X, Diaz LA, Fairley JA, et al. The anti-desmoglein 1 autoantibodies in pemphigus vulgaris sera are pathogenic. J Invest Dermatol 1999;112:739-743.

30 Kamisawa T. IgG4-positive plasma cells specifically infiltrate various organs in autoimmune pancreatitis. Pancreas 2004;29:167-168.

31 Yadav D, Notahara K, Smyrk TC, et al. Idiopathic tumefactive chronic pancreatitis: clinical profile, histology, and natural history after resection. Clin Gastroenterol Hepatol 2003;1:129-135. 